Honam Mathematical J. 34 (2012), No. 1, pp. 125-133

http://dx.doi.org/10.5831/HMJ.2012.34.1.125

\title{
CHARACTERIZATIONS OF FALLING FUZZY IDEALS
}

\author{
Young Bae Jun, Seok-Zun Song* and Eun Hwan Roh
}

\begin{abstract}
Relations among falling fuzzy ideals, falling fuzzy implicative ideals, falling fuzzy positive implicative ideals and falling fuzzy commutative ideals are considered. Characterizations of falling fuzzy positive implicative ideals and other related ideals are discussed.
\end{abstract}

\section{Introduction}

In the study of a unified treatment of uncertainty modelled by means of combining probability and fuzzy set theory, Goodman [1] pointed out the equivalence of a fuzzy set and a class of random sets. Wang and Sanchez [14] introduced the theory of falling shadows which directly relates probability concepts with the membership function of fuzzy sets. Falling shadow representation theory shows us the way of selection laid on the joint degrees distributions. It is reasonable and convenient approach for the theoretical development and the practical applications of fuzzy sets and fuzzy logics. The mathematical structure of the theory of falling shadows is formulated in [13]. Tan et al. [11, 12] established a theoretical approach to define a fuzzy inference relation and fuzzy set operations based on the theory of falling shadows. Jun and Park [6] discussed the notion of a falling fuzzy subalgebra/ideal of a BCK/BCIalgebra. Jun et al. [3, 4] also considered falling fuzzy positive implicativeideals and falling fuzzy commutative ideals. In this paper, we establish a theoretical approach to define a fuzzy implicative ideal in a $B C K$-algebra based on the theory of falling shadows. We consider relations among falling fuzzy ideals, falling fuzzy implicative ideals, falling

Received February 28, 2012. Accepted March 14, 2012.

2000 Mathematics Subject Classification. 06F35, 03G25, 08 A72.

Key words and phrases. Falling shadow, Positive implicative ideal, Fuzzy positive implicative ideal, Falling fuzzy positive implicative ideal.

*Corresponding author. 
fuzzy positive implicative ideals and falling fuzzy commutative ideals. We deal with characterizations of falling fuzzy (commutative, positive implicative, implicative) ideals.

\section{Preliminaries}

A $B C K / B C I$-algebra is an important class of logical algebras introduced by K. Iséki and was extensively investigated by several researchers.

An algebra $(X ; *, 0)$ of type $(2,0)$ is called a $B C I$-algebra if it satisfies the following conditions:

(I) $(\forall x, y, z \in X)(((x * y) *(x * z)) *(z * y)=0)$,

(II) $(\forall x, y \in X)((x *(x * y)) * y=0)$,

(III) $(\forall x \in X)(x * x=0)$,

(IV) $(\forall x, y \in X)(x * y=0, y * x=0 \Rightarrow x=y)$.

If a BCI-algebra $X$ satisfies the following identity:

(V) $(\forall x \in X)(0 * x=0)$,

then $X$ is called a $B C K$-algebra. Any BCK/BCI-algebra $X$ satisfies the following axioms:

(a1) $(\forall x \in X)(x * 0=x)$,

(a2) $(\forall x, y, z \in X)(x \leq y \Rightarrow x * z \leq y * z, z * y \leq z * x)$,

(a3) $(\forall x, y, z \in X)((x * y) * z=(x * z) * y)$,

where $x \leq y$ if and only if $x * y=0$.

A subset $I$ of a BCK/BCI-algebra $X$ is called an ideal of $X$ if it satisfies:

(b1) $0 \in I$.

(b2) $(\forall x \in X)(\forall y \in I)(x * y \in I \Longrightarrow x \in I)$.

Every ideal $I$ of a BCK/BCI-algebra $X$ has the following assertion:

$$
(\forall x \in X)(\forall y \in I)(x \leq y \Longrightarrow x \in I) .
$$

A subset $I$ of a $B C K$-algebra $X$ is called a positive implicative ideal of $X$ if it satisfies (b1) and

(b3) $(\forall x, y, z \in X)((x * y) * z \in I, y * z \in I \Rightarrow x * z \in I)$.

A subset $I$ of a $B C K$-algebra $X$ is called a commutative ideal of $X$ if it satisfies (b1) and

(b4) $(\forall x, y, z \in X)((x * y) * z \in I, z \in I \Rightarrow x *(y *(y * x)) \in I)$.

A subset $I$ of a $B C K$-algebra $X$ is called an implicative ideal of $X$ if it satisfies (b1) and

(b5) $(\forall x, y, z \in X)((x *(y * x)) * z \in I, z \in I \Rightarrow x \in I)$. 
We refer the reader to the book [9] for further information regarding $B C K$-algebras.

A fuzzy set $\mu$ in a $B C K / B C I$-algebra $X$ is called a fuzzy ideal of $X$ (see [15]) if it satisfies:

(c1) $(\forall x \in X)(\mu(0) \geq \mu(x))$.

(c2) $(\forall x, y \in X)(\mu(x) \geq \min \{\mu(x * y), \mu(y)\})$.

A fuzzy set $\mu$ in a $B C K$-algebra $X$ is called a fuzzy positive implicativeideal of $X$ (see [2]) if it satisfies (c1) and

(c3) $(\forall x, y, z \in X)(\mu(x * z) \geq \min \{\mu((x * y) * z), \mu(y * z)\})$.

A fuzzy set $\mu$ in a $B C K$-algebra $X$ is called a fuzzy commutative ideal of $X$ (see [8]) if it satisfies (c1) and

(c4) $(\forall x, y, z \in X)(\mu(x *(y *(y * x))) \geq \min \{\mu((x * y) * z), \mu(z)\})$.

A fuzzy set $\mu$ in a $B C K$-algebra $X$ is called a fuzzy implicative ideal of $X$ (see [10]) if it satisfies (c1) and

(c5) $(\forall x, y, z \in X)(\mu(x) \geq \min \{\mu((x *(y * x)) * z), \mu(z)\})$.

We now display the basic theory on falling shadows. We refer the reader to the papers $[1,11,12,13,14]$ for further information regarding falling shadows.

Given a universe of discourse $U$, let $\mathbb{P}(U)$ denote the power set of $U$. For each $u \in U$, let

$$
\dot{u}:=\{E \mid u \in E \text { and } E \subseteq U\},
$$

and for each $E \in \mathbb{P}(U)$, let

$$
\dot{E}:=\{\dot{u} \mid u \in E\} .
$$

An ordered pair $(\mathbb{P}(U), \mathbb{B})$ is said to be a hyper-measurable structure on $U$ if $\mathbb{B}$ is a $\sigma$-field in $\mathbb{P}(U)$ and $\dot{U} \subseteq \mathbb{B}$. Given a probability space $(\Omega, \mathbb{A}, P)$ and a hyper-measurable structure $(\mathbb{P}(U), \mathbb{B})$ on $U$, a random set on $U$ is defined to be a mapping $\xi: \Omega \rightarrow \mathbb{P}(U)$ which is $\mathbb{A}-\mathbb{B}$ measurable, that is,

$$
(\forall C \in \mathbb{B})\left(\xi^{-1}(C)=\{\omega \mid \omega \in \Omega \text { and } \xi(\omega) \in C\} \in \mathbb{A}\right) .
$$

Suppose that $\xi$ is a random set on $U$. Let

$$
\tilde{H}(u):=P(\omega \mid u \in \xi(\omega)) \text { for each } u \in U .
$$

Then $\tilde{H}$ is a kind of fuzzy set in $U$. We call $\tilde{H}$ a falling shadow of the random set $\xi$, and $\xi$ is called a cloud of $\tilde{H}$.

For example, $(\Omega, \mathbb{A}, P)=([0,1], \mathbb{A}, m)$, where $\mathbb{A}$ is a Borel field on $[0,1]$ and $m$ is the usual Lebesgue measure. Let $\tilde{H}$ be a fuzzy set in $U$ 
and $\tilde{H}_{t}:=\{u \in U \mid \tilde{H}(u) \geq t\}$ be a $t$-cut of $\tilde{H}$. Then

$$
\xi:[0,1] \rightarrow \mathbb{P}(U), t \mapsto \tilde{H}_{t}
$$

is a random set and $\xi$ is a cloud of $\tilde{H}$. We shall call $\xi$ defined above as the cut-cloud of $\tilde{H}$ (see [1]).

\section{Properties of falling fuzzy ideals}

In what follows let $X$ denote a $B C K$-algebra unless otherwise specified.

Definition $3.1([3])$. Let $(\Omega, \mathbb{A}, P)$ be a probability space, and let

$$
\xi: \Omega \rightarrow \mathbb{P}(X)
$$

be a random set. If $\xi(\omega)$ is a (positive implicative, commutative, implicative) ideal of $X$ for any $\omega \in \Omega$, then the falling shadow $\tilde{H}$ of the random set $\xi$, i.e.,

$$
\tilde{H}(x)=P(\omega \mid x \in \xi(\omega))
$$

is called a falling fuzzy (positive implicative, commutative, implicative) ideal of $X$.

Let $(\Omega, \mathbb{A}, P)$ be a probability space and let

$$
F(X):=\{f \mid f: \Omega \rightarrow X \text { is a mapping }\} .
$$

Define an operation $\diamond$ on $F(X)$ by

$$
(\forall \omega \in \Omega)((f \diamond g)(\omega)=f(\omega) * g(\omega))
$$

for all $f, g \in F(X)$. Let $\theta \in F(X)$ be defined by $\theta(\omega)=0$ for all $\omega \in \Omega$. Then $(F(X) ; \diamond, \theta)$ is a $B C K$-algebra (see $[6])$.

Let $(\Omega, \mathbb{A}, P)$ be a probability space and $\tilde{H}$ a falling shadow of a random set $\xi: \Omega \rightarrow \mathbb{P}(X)$. For any $x \in X$, let

$$
\Omega(x ; \xi):=\{\omega \in \Omega \mid x \in \xi(\omega)\} .
$$

Then $\Omega(x ; \xi) \in \mathbb{A}$.

Lemma $3.2([4])$. Every falling fuzzy commutative ideal is a falling fuzzy ideal.

Lemma $3.3([4])$. If a falling shadow $\tilde{H}$ of a random set $\xi: \Omega \rightarrow$ $\mathbb{P}(X)$ is a falling fuzzy commutative ideal of $X$, then

$$
(\forall x, y, z \in X)(\Omega((x * y) * z ; \xi) \cap \Omega(z ; \xi) \subseteq \Omega(x *(y *(y * x)) ; \xi)) .
$$


Theorem 3.4. Let $\tilde{H}$ be a falling shadow of a random set $\xi: \Omega \rightarrow$ $\mathbb{P}(X)$. Then $\tilde{H}$ is a falling fuzzy commutative ideal of $X$ if and only if $\tilde{H}$ is a falling fuzzy ideal of $X$ that satisfies the condition (3.3).

Proof. Necessity follows from Lemmas 3.2 and 3.3. Assume that $\tilde{H}$ is a falling fuzzy ideal of $X$ that satisfies the condition (3.3). Let $\omega \in \Omega$ and $x, y, z \in X$ be such that $z \in \xi(\omega)$ and $(x * y) * z \in \xi(\omega)$. Using (3.3), we have

$$
\omega \in \Omega((x * y) * z ; \xi) \cap \Omega(z ; \xi) \subseteq \Omega(x *(y *(y * x)) ; \xi),
$$

and so $x *(y *(y * x)) \in \xi(\omega)$. Therefore $\tilde{H}$ is a falling fuzzy commutative ideal of $X$.

Theorem 3.5. Let $\tilde{H}$ be a falling shadow of a random set $\xi: \Omega \rightarrow$ $\mathbb{P}(X)$. Then $\tilde{H}$ is a falling fuzzy commutative ideal of $X$ if and only if $\tilde{H}$ is a falling fuzzy ideal of $X$ satisfying the condition

$$
(\forall x, y \in X)(\Omega(x * y ; \xi) \subseteq \Omega(x *(y *(y * x)) ; \xi)) .
$$

Proof. Let $\tilde{H}$ be a falling fuzzy commutative ideal of $X$. Then $\tilde{H}$ is a falling fuzzy ideal of $X$ by Lemma 3.2. Let $\omega \in \Omega(x * y ; \xi)$. Then $(x * y) * 0=x * y \in \xi(\omega)$. Since $0 \in \xi(\omega)$ and $\xi(\omega)$ is a commutative ideal of $X$, it follows from (b4) that $x *(y *(y * x)) \in \xi(\omega)$ so that $\omega \in \Omega(x *(y *(y * x)) ; \xi)$. Hence

$$
\Omega(x * y ; \xi) \subseteq \Omega(x *(y *(y * x)) ; \xi)
$$

for all $x, y \in X$.

Conversely, let $\tilde{H}$ be a falling fuzzy ideal of $X$ that satisfies the condition (3.4). Let $\omega \in \Omega$ and $x, y, z \in X$ be such that $(x * y) * z \in \xi(\omega)$ and $z \in \xi(\omega)$. Since $\xi(\omega)$ is an ideal of $X$, it follows that $x * y \in \xi(\omega)$ so from (3.4) that

$$
\omega \in \Omega(x * y ; \xi) \subseteq \Omega(x *(y *(y * x)) ; \xi) .
$$

Hence $x *(y *(y * x)) \in \xi(\omega)$, and therefore $\tilde{H}$ is a falling fuzzy commutative ideal of $X$.

Lemma 3.6 ([5]). Every falling fuzzy implicative ideal is a falling fuzzy ideal.

Lemma 3.7 ([5]). If a falling shadow $\tilde{H}$ of a random set $\xi: \Omega \rightarrow$ $\mathbb{P}(X)$ is a falling fuzzy implicative ideal of $X$, then

$$
(\forall x, y, z \in X)(\Omega((x *(y * x)) * z ; \xi) \cap \Omega(z ; \xi) \subseteq \Omega(x ; \xi)) .
$$


Theorem 3.8. Let $\tilde{H}$ be a falling shadow of a random set $\xi: \Omega \rightarrow$ $\mathbb{P}(X)$. Then $\tilde{H}$ is a falling fuzzy implicative ideal of $X$ if and only if $\tilde{H}$ is a falling fuzzy ideal of $X$ that satisfies the condition (3.5).

Proof. The necessity is induced by Lemmas 3.6 and 3.7. Assume that $\tilde{H}$ is a falling fuzzy ideal of $X$ that satisfies the condition (3.5). Let $\omega \in \Omega$ and $x, y, z \in X$ be such that $z \in \xi(\omega)$ and $(x *(y * x)) * z \in \xi(\omega)$. Then

$$
\omega \in \Omega((x *(y * x)) * z ; \xi) \cap \Omega(z ; \xi) \subseteq \Omega(x ; \xi)
$$

by (3.5), and so $x \in \xi(\omega)$. Thus $\tilde{H}$ is a falling fuzzy implicative ideal of $X$.

Note that every falling fuzzy implicative ideal is a falling fuzzy (positive implicative) ideal, but the converse is not true (see [5]). Now we provide conditions for a falling fuzzy (positive implicative) ideal to be a falling fuzzy implicative ideal.

Theorem 3.9. Let $\tilde{H}$ be a falling shadow of a random set $\xi: \Omega \rightarrow$ $\mathbb{P}(X)$. If $\tilde{H}$ is a falling fuzzy ideal of $X$ that satisfies the following condition:

$$
(\forall x, y \in X)(\Omega(x *(y * x) ; \xi) \subseteq \Omega(x ; \xi)),
$$

then $\tilde{H}$ is a falling fuzzy implicative ideal of $X$.

Proof. Let $\omega \in \Omega$ and $x, y, z \in X$ be such that $z \in \xi(\omega)$ and $(x *$ $(y * x)) * z \in \xi(\omega)$. Since $\xi(\omega)$ is an ideal of $X$, it follows from (b2) that $x *(y * x) \in \xi(\omega)$ so from (3.6) that $\omega \in \Omega(x *(y * x) ; \xi) \subseteq \Omega(x ; \xi)$. Hence $x \in \xi(\omega)$, and consequently $\tilde{H}$ is a falling fuzzy implicative ideal of $X$.

Lemma 3.10 ([7]). Every falling fuzzy positive implicativeideal $\tilde{H}$ of $X$ satisfies the following condition:

$$
(\forall x, y \in X)(\Omega((x * y) * y ; \xi) \subseteq \Omega(x * y ; \xi)) .
$$

Theorem 3.11. Let $\tilde{H}$ be a falling shadow of a random set $\xi: \Omega \rightarrow$ $\mathbb{P}(X)$. Then $\tilde{H}$ is a falling fuzzy implicative ideal of $X$ if and only if $\tilde{H}$ is a falling fuzzy positive implicativeideal of $X$ that satisfies the condition:

$$
(\forall x, y \in X)(\Omega(y *(y * x) ; \xi) \subseteq \Omega(x *(x * y) ; \xi)) .
$$


Proof. Let $\tilde{H}$ be a falling fuzzy implicative ideal of $X$. Then $\tilde{H}$ is a falling fuzzy positive implicativeideal of $X$ (see [5, Theorem 2.8]). Let $\omega \in \Omega$ and $x, y \in X$ be such that $\omega \in \Omega(y *(y * x) ; \xi)$. Then

$$
\begin{aligned}
& (x *(x * y)) *(y *(x *(x * y))) \leq(x *(x * y)) *(y * x) \\
& =(x *(y * x)) *(x * y) \leq y *(y * x) \in \xi(\omega) .
\end{aligned}
$$

Since $\xi(\omega)$ is an implicative ideal and hence an ideal of $X$, it follows from (a1) and (2.1) that

$((x *(x * y)) *(y *(x *(x * y)))) * 0=(x *(x * y)) *(y *(x *(x * y))) \in \xi(\omega)$ so that $x *(x * y) \in \xi(\omega)$, that is, $\omega \in \Omega(x *(x * y) ; \xi)$. Hence (3.8) is valid.

Conversely, suppose that $\tilde{H}$ is a falling fuzzy positive implicativeideal of $X$ satisfying the condition (3.8). Let $\omega \in \Omega$ and $x, y, z \in X$ be such that $z \in \xi(\omega)$ and $(x *(y * x)) * z \in \xi(\omega)$. Since $\xi(\omega)$ is a positive implicativeideal and hence an ideal of $X$, it follows from (I) and (b2) that

$$
(y *(y * x)) *(y * x) \leq x *(y * x) \in \xi(\omega)
$$

so that $(y *(y * x)) *(y * x) \in \xi(\omega)$. Using Lemma 3.10 and (3.8), we get

$$
\begin{aligned}
\omega & \in \Omega((y *(y * x)) *(y * x) ; \xi) \\
& \subseteq \Omega(y *(y * x) ; \xi) \subseteq \Omega(x *(x * y) ; \xi),
\end{aligned}
$$

and so $x *(x * y) \in \xi(\omega)$. Note that

$$
(x * y) * z \leq x * y \leq x *(y * x) \in \xi(\omega),
$$

and hence $(x * y) * z \in \xi(\omega)$ by (2.1). Since $z \in \xi(\omega)$ and $\xi(\omega)$ is an ideal of $X$, it follows that $x * y \in \xi(\omega)$ so that $x \in \xi(\omega)$. Therefore $\tilde{H}$ is a falling fuzzy implicative ideal of $X$.

Theorem 3.12. Let $\tilde{H}$ be a falling shadow of a random set $\xi: \Omega \rightarrow$ $\mathbb{P}(X)$. Then $\tilde{H}$ is a falling fuzzy implicative ideal of $X$ if and only if it is both a falling fuzzy positive implicativeideal and a falling fuzzy commutative ideal of $X$.

Proof. Necessity follows from [5, Theorem 2.8]. Conversely let $\tilde{H}$ be both a falling fuzzy positive implicativeideal and a falling fuzzy commutative ideal of $X$. Then $\tilde{H}$ is a falling fuzzy ideal of $X$, and so it is sufficient to show that the condition (3.6). Let $\omega \in \Omega(x *(y * x) ; \xi)$ for all $\omega \in \Omega$ and $x, y \in X$. Then

$$
(y *(y * x)) *(y * x) \leq x *(y * x) \in \xi(\omega),
$$


and thus $(y *(y * x)) *(y * x) \in \xi(\omega)$ by (2.1). Using Lemma 3.10, we have

$$
\omega \in \Omega((y *(y * x)) *(y * x) ; \xi) \subseteq \Omega(y *(y * x) ; \xi) .
$$

Since $x * y \leq x *(y * x)$, we obtain $x * y \in \xi(\omega)$, which implies from Theorem 3.5 that

$$
\omega \in \Omega(x * y ; \xi) \subseteq \Omega(x *(y *(y * x)) ; \xi),
$$

that is, $x *(y *(y * x)) \in \xi(\omega)$. Since $y *(y * x) \in \xi(\omega)$ and $\xi(\omega)$ is an ideal of $X$, we have $x \in \xi(\omega)$, i.e., $\omega \in \Omega(x ; \xi)$. Therefore $\Omega(x *(y * x) ; \xi) \subseteq$ $\Omega(x ; \xi)$ for all $x, y \in X$. According to Theorem $3.9, \tilde{H}$ is a falling fuzzy implicative ideal of $X$.

\section{References}

[1] I. R. Goodman, Fuzzy sets as equivalence classes of random sets, in "Recent Developments in Fuzzy Sets and Possibility Theory"(R. Yager, Ed.), Pergamon, New York (1982).

[2] Y. B. Jun, S. M. Hong, J. Meng and X. L. Xin, Characterizations of fuzzy positive implicative ideals in BCK-algebras, Math. Japon. 40 (1994) 503-507.

[3] Y. B. Jun and M. S. Kang, Fuzzy positive implicative ideals of BCK-algebras based on the theory of falling shadows, Comput. Math. Appl. 61 (2011) 62-67.

[4] Y. B. Jun and M. S. Kang, Falling fuzzy commutative ideals of BCK-algebras, J. Korean Soc. Math. Edu. Ser. B: Pure Appl. Math. 18 (2011) 41-50.

[5] Y. B. Jun, M. S. Kang and C. H. Park, Implicative ideals of BCK-algebras based on the fuzzy sets and the theory of falling shadows, Internat. J. Math. Math. Sci. Volume 2010, Article ID 819463, 11 pages.

[6] Y. B. Jun and C. H. Park, Falling shadows applied to subalgebras and ideals of $B C K / B C I$-algebras, Chaos, Solitions and Fractals (submitted).

[7] Y. B. Jun, M. A. Öztürk and A. Firat, Characterizations of falling fuzzy positive implicative ideals in BCK-algebras, Appl. Soft Comput. (submitted).

[8] Y. B. Jun and E. H. Roh, Fuzzy commutative ideals of BCK-algebras, Fuzzy Sets and Systems 64 (1994) 401-405.

[9] J. Meng and Y. B. Jun, BCK-algebras, Kyungmoon Sa Co. Seoul 1994.

[10] S. M. Mostafa, Fuzzy implicative ideals of BCK-algebras, Fuzzy Sets and Systems 87 (1997) 361-368.

[11] S. K. Tan, P. Z. Wang and E. S. Lee, Fuzzy set operations based on the theory of falling shadows, J. Math. Anal. Appl. 174 (1993) 242-255.

[12] S. K. Tan, P. Z. Wang and X. Z. Zhang, Fuzzy inference relation based on the theory of falling shadows, Fuzzy Sets and Systems 53 (1993) 179-188.

[13] P. Z. Wang, Fuzzy Sets and Falling Shadows of Random Sets, Beijing Normal Univ. Press, People's Republic of China, 1985. [In Chinese]

[14] P. Z. Wang and E. Sanchez, Treating a fuzzy subset as a projectable random set, in: M. M. Gupta, E. Sanchez, Eds., "Fuzzy Information and Decision" (Pergamon, New York, 1982) 212-219.

[15] O. G. Xi, Fuzzy BCK-algebras, Math. Japon. 36 (1991) 935-942. 
Young Bae Jun

Department of Mathematics Education (and RINS), Gyeongsang National University,

Chinju 660-701, Korea.

E-mail: skywine@gmail.com

\section{Seok-Zun Song}

Department of Mathematics, Jeju National University,

Jeju 690-756, Korea.

E-mail: skywine@gmail.com

\section{Eun Hwan Roh}

Department of Mathematics Education, Chinju National University of Education,

Chinju 660-756, Korea.

E-mail: skywine@gmail.com 